\title{
Análisis del Convertidor CA/CD/CA (Back to Back) para la Gestión del Flujo de Potencia
}

\author{
Janeth Alcalá, Saida Charre, Miguel Durán, Jorge Gudiño \\ Facultad de Ingeniería Electromecánica, Universidad de Colima, Colima-México \\ (e-mail: janethalcala@ucol.mx, scharre@ucol.mx,mduran@ucol.mx, jglau@ucol.mx)
}

Recibido Mar. 19, 2014; Aceptado Jun. 13, 2014; Versión final recibida Ago. 18, 2014

\begin{abstract}
Resumen
Este artículo presenta una solución basada en el convertidor CA/CD/CA (Back to Back, BTB) para la gestión del flujo de potencia entre dos sistemas interconectados de corriente alterna. El objetivo es mostrar las ventajas que presenta el convertidor BTB para la transferencia de potencia activa y de potencia reactiva entre dos sistemas de corriente alterna interconectados. Mediante una estrategia de control lineal es posible desacoplar los términos activos y reactivos de corriente y controlar de forma independiente la potencia activa y reactiva. El control se desarrolla en el marco de referencia síncrono de cuadratura directa (DQ) asumiendo un sistema trifásico balanceado. Los resultados de simulación se evalúan en un sistema de 3kVA y para las pruebas experimentales se utiliza un prototipo de laboratorio de la misma capacidad.
\end{abstract}

Palabras clave: convertidor $C A / C D / C A$, flujo de potencia bidireccional, control lineal

\section{Analysis of the Back to Back converter for power flow management}

\begin{abstract}
This paper discusses a Back to Back (BTB) power converter based solution for the management of power flow between two interconnected AC systems. The main objective of this work is to show the advantages of the BTB converter to manage the active and reactive power between two interconnected AC systems. The active and reactive current components are decoupled through a linear control strategy allowing the active and reactive powers are independently controlled. The control strategy is developed on the directquadrature synchronous reference frame (DQ) considering a balanced three-phase three wire system. The performance of the BTB converter for power flow control is demonstrated through simulation and experimental results using a 3kVA power converter.
\end{abstract}

Keywords: back-to-back converter, bidirectional power flow, linear control 


\section{INTRODUCCIÓN}

La gestión del flujo de potencia en aplicaciones que interconectan dos sistemas de CA es un tema que ha cobrado relevancia en los últimos años, especialmente en el campo de las energías emergentes (sistemas de generación eólicos, fotovoltaicos, microredes, etc), ya que sistemas han impuestos nuevos retos a los esquemas reportados en el pasado para el manejo del flujo de potencia (Gallego-Gómez, et al., 2014, Guacaneme et al., 2014), en donde las redes eléctricas se podían controlar con sistemas relativamente simples, los flujos de potencia eran exclusivamente unidireccionales y los sistemas operaban dentro de los límites establecidos. Sin embargo, en las últimas décadas, la creciente penetración de la generación distribuida, asociada fundamentalmente a las energías renovables, y la aparición de los flujos bidireccionales, han desencadenado un proceso de cambio radical de las redes eléctricas, por lo que ha sido necesario instalar múltiples dispositivos de medición y/o de control con el objetivo de mejorar el rendimiento de las redes, reducir las pérdidas y dotar de una mayor flexibilidad al sistema de transporte de energía para mantener su seguridad y confiabilidad de operación dentro de los rangos deseados. Esto en conjunto ha originado que en la actualidad, tanto la comunidad científica como la industrial evalúen nuevas estructuras de potencia y estrategias de control que cumplan con las expectativas de operación requeridas en sistemas donde la gestión del flujo de potencia es fundamental.

En este sentido, el convertidor de potencia Back to Back (BTB) basado en convertidores tipo fuente de tensión (VSC) es un método típicamente usado para procesar eficazmente el flujo de potencia en sistemas de CA interconectados, ya que permite controlar de manera independiente tanto el flujo bidireccional de potencia activa como de potencia reactiva, desde baja potencia (Majumder et al., 2010) hasta alta potencia (Saeedifard y Iravani 2010), en procesos y aplicaciones industriales que incluyen desde control de motores, generadores eólicos y balance de carga entre alimentadores, hasta sistemas de generación distribuida, por mencionar algunos. De aquí que el uso del convertidor BTB modulado mediante técnicas PWM (Pulse Width Modulation) se ha incrementado en los últimos años.

Respecto al estudio del convertidor BTB se ha desarrollado una gran cantidad de trabajo de acuerdo a problemáticas asociadas a aplicaciones específicas (Weihao et al., 2009). Sin embargo, en la literatura no se encuentra reportado un estudio que permita identificar adecuadamente las limitantes físicas que presenta este convertidor para el manejo del flujo de potencia. Además, aun cuando en la mayoría de los trabajos reportados se subraya que el convertidor BTB es una opción atractiva para el control bidireccional del flujo de potencia entre dos sistemas de CA (Liming Liu et al., 2007), los resultados presentados no permiten evaluar el desempeño del convertidor en ambos lados de CA.

En este artículo se analiza el desempeño del convertidor BTB trifásico para gestionar el flujo de potencia entre dos sistemas de CA. El análisis se desarrolla en el marco de referencia síncrono DQ. El estudio se enfoca en analizar la capacidad que tiene el convertidor BTB para procesar potencia, especialmente ante condiciones que obliguen al convertidor a invertir el sentido del flujo de potencia activa y a cambiar de una región inductiva a una capacitiva en el caso de la potencia reactiva. Para el control del flujo de potencia se considera uno de los esquemas de control más utilizado en aplicaciones para VSC que ha demostrado un buen desempeño al desacoplar las componentes activa (componente directa, d) y reactiva (componente en cuadratura, q) de los sistemas de CA (Zeliang Shu, et. al., 2011) y se conoce como control desacoplado de potencia. En las siguientes secciones se presenta la topología de potencia, el principio de operación, el esquema de control y los resultados de simulación y experimentales representativos. Para las pruebas experimentales se utiliza un prototipo de 3 kVAR.

\section{EL CONVERTIDOR BACK TO BACK}

El convertidor BTB está formado por convertidores VSC, como se muestra en la Fig. 1; el convertidor del lado de la red eléctrica es $\mathrm{VSC}_{1}$ y el convertidor del lado de la carga es $\mathrm{VSC}_{2}$. La carga puede ser pasiva, activa u otra red eléctrica. VSC y $\mathrm{VSC}_{2}$ comparten el bus de corriente directa (CD) a través del capacitor $\mathrm{C}_{c d}$, el cual permite que ambos VSC se puedan controlar de manera independiente en cierto rango de potencia.VSC $\mathrm{V}_{1} \mathrm{VSC}_{2}$ puede actuar como un rectificador o un inversor en función de la dirección del flujo de potencia. Las flechas bidireccionales se utilizan para indicar que el flujo de potencia activa puede cambiar en cualquier momento e ir en cualquier dirección y que la potencia reactiva puede se controla entre VSC $_{1}$ y la red eléctrica o $\mathrm{VSC}_{2}$ y la carga, puede ser capacitiva o inductiva.La operación fundamental del convertidor BTB se explica al considerar VSC 1 y VSC 2 como dos convertidores desacoplados en los cuales la amplitud, fase y frecuencia se pueden controlar de forma independiente el uno del otro. El análisis se presenta para el caso particular de $\mathrm{VSC}_{1}$ y se utiliza el mismo procedimiento para $\mathrm{VSC}_{2}$. En la Fig. 2(a) se muestra un esquema equivalente por fase del convertidor BTB trifásico y en la Fig. 2(b) el circuito equivalente. 


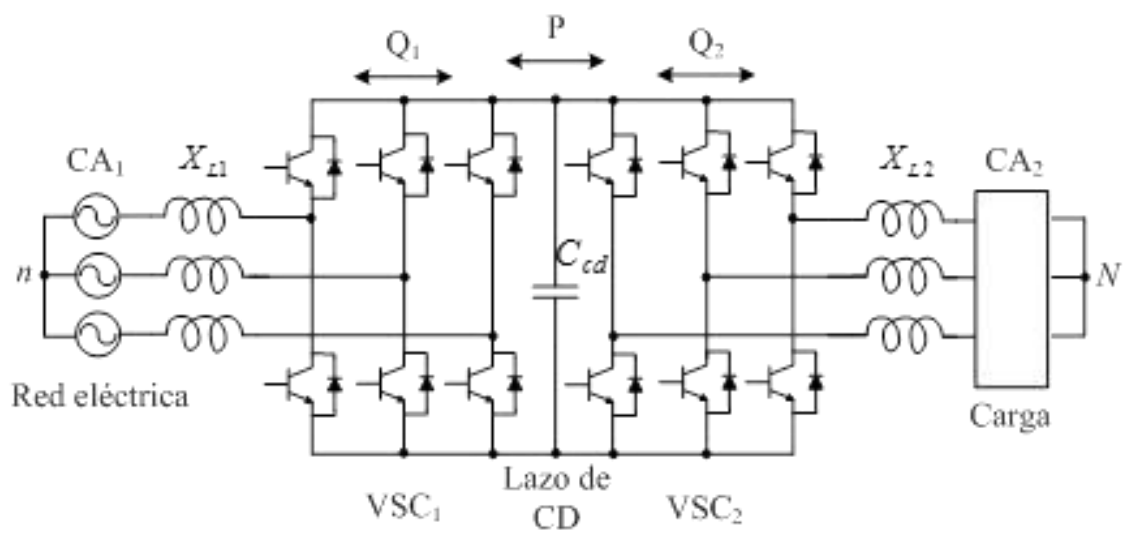

Fig. 1: Convertidor BTB.

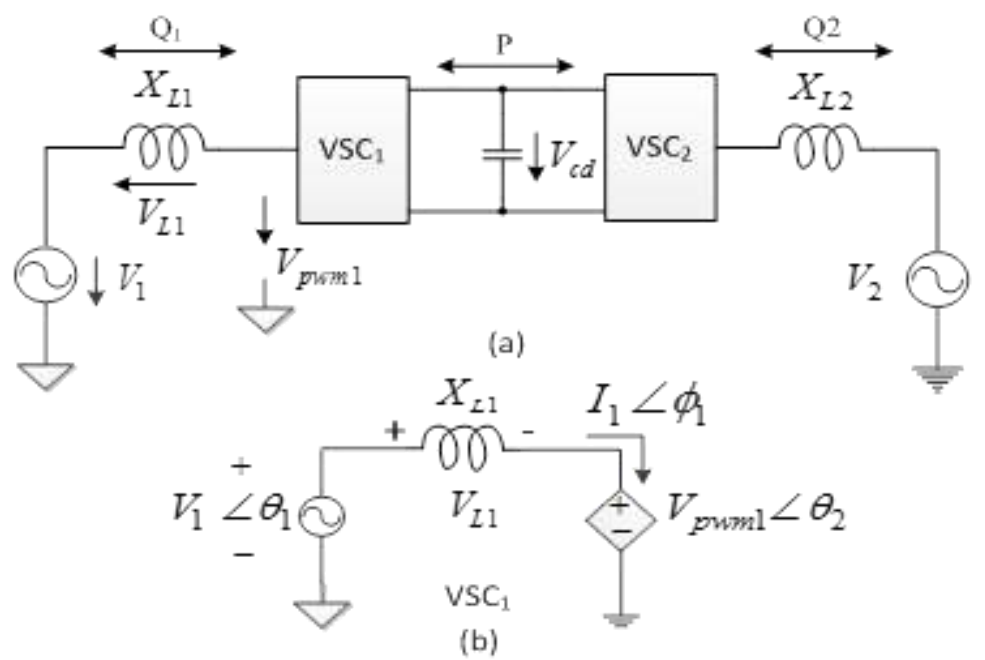

Fig. 2: Representación equivalente por fase del convertidor BTB.

La fuente controlada de tensión $V_{p w m 1}$ representa la tensión generada en las terminales del convertidor VSC 1 y es proporcional a la tensión de CD, así como la señal de modulación PWM. La potencia aparente (S) suministrada por la fuente de energía se calcula considerando una reactancia de línea sin pérdidas y está dada por:

$$
S_{1}=\frac{V_{1} V_{p w m 1} \operatorname{sen}\left(\theta_{1}-\theta_{2}\right)}{\omega_{1} L_{1}}+j \frac{V_{1}^{2}-V_{1} V_{p w m 1} \cos \left(\theta_{1}-\theta_{2}\right)}{\omega_{1} L_{1}}
$$

donde $V_{1}$ y $\theta_{1}$ son la magnitud y la fase de la fuente de alimentación; $V_{p w m 1}$ y $\theta_{2}$ son la magnitud y la fase de la fuente de tensión controlada y $\omega 1$ representa la frecuencia de línea. Como resultado, la caída de tensión $\left(V_{L 1}\right)$ a través del reactor $\left(X_{L 1}\right)$ se puede variar para controlar la potencia activa y la potencia reactiva. De (1) se observa que la potencia activa $(P)$ suministrada se da como una función de: 1) la magnitud de la tensión de fase instantánea $V_{1}$ y $\left.V_{p w m 1}, 2\right)$ el desfase entre $V_{1}$ y $V_{p w m 1}$ y 3) la reactancia de enlace.

El flujo de potencia activa $\left(P_{1}\right)$ entre $V_{S C}$ y $V_{1}$ es proporcional a la tensión y a la corriente de CD y se puede controlar cambiando el ángulo de fase $\left(\theta_{1}-\theta_{2}\right)$ entre la tensión a frecuencia fundamental generada por el convertidor $\left(V_{p w m 1}\right)$ y la tensión en el bus de CD $\left(V_{c d}\right)$, $\mathrm{P}_{1}$ se calcula como:

$P_{1}=\frac{V_{1} V_{p w m 1} \sin \left(\theta_{1}-\theta_{2}\right)}{\omega_{1} L_{1}}$

El flujo de potencia reactiva $\left(Q_{1}\right)$ se determina por la diferencia de las magnitudes de la tensión de CA y la tensión fundamental generada en las terminales del convertidor. El flujo de potencia reactiva se calcula de acuerdo a: 
$Q_{1}=\frac{V_{1}^{2}-V_{1} V_{p w m 1} \cos \left(\theta_{1}-\theta_{2}\right)}{\omega_{1} L_{1}}$

La potencia activa y la reactiva se definen como positivas si la potencia fluye de la red eléctrica a la carga y el desplazamiento del ángulo de fase $\left(\theta_{1}-\theta_{2}\right)$ como positivo si la tensión de salida del convertidor está en atraso respecto a la tensión de la red eléctrica.

\section{MODELADO DEL SISTEMA Y REQUERIMIENTOS DE OPERACIÓN}

Para obtener el modelo matemático del convertidor BTB se utiliza la transformación DQ trifásica y se asume un sistema trifásico balanceado en conexión delta, y cada transformación se relaciona con el lado de CA correspondiente. La transformación DQ permite que el modelo matemático del convertidor BTB se pueda expresar mediante dos variables con componentes en CD que proporcionan información de la amplitud y la fase. Como resultado, a través del modelo DQ los objetivos de control se simplifican al convertir el problema de seguimiento de una referencia sinusoidal en un problema de regulación.

El modelo matemático DQ para el convertidor BTB está dado por:

$$
\begin{aligned}
& \frac{d i_{1}^{d}}{d t}=-\frac{R_{1}}{L_{1}} i_{1}^{d}+\omega_{1} i_{1}^{q}+\frac{1}{L_{1}} v_{1}^{d}-\frac{1}{2 U_{T 1} L_{1}} v_{c d} m_{1}^{d} \\
& \frac{d i_{1}^{q}}{d t}=-\frac{R_{1}}{L_{1}} i_{1}^{q}-\omega_{1} i_{1}^{d}-\frac{1}{2 U_{T 1} L_{1}} v_{c d} m_{1}^{q} \\
& \frac{d i_{2}^{d}}{d t}=-\frac{R_{2}}{L_{2}} i_{2}^{d}+\omega_{2} i_{2}^{q}+\frac{1}{L_{2}} v_{2}^{d}-\frac{1}{2 U_{T 2} L_{2}} v_{c d} m_{2}^{d} \\
& \frac{d i_{2}^{q}}{d t}=-\frac{R_{2}}{L_{2}} i_{2}^{q}-\omega_{2} i_{2}^{d}-\frac{1}{2 U_{T 2} L_{2}} v_{c d} m_{2}^{q} \\
& C_{c d} \frac{d v_{c d}}{d t}=\frac{3}{4}\left[\frac{1}{U_{T 1}}\left(m_{1}^{d} i_{1}^{d}+m_{1}^{q} i_{1}^{q}\right)+\frac{1}{U_{T 2}}\left(m_{2}^{d} i_{2}^{d}+m_{2}^{q} i_{2}^{q}\right)\right]
\end{aligned}
$$

donde los sub índices 1 y 2 se asocian a los convertidores VSC y VSC $_{2}$ respectivamente; $R_{1,2}$ representa la resistencia asociada a las reactancias, los términos moduladores $m_{l, 2}^{d, q}$ son las entradas de control y $U_{T 1,2}$ son las amplitudes pico de las portadoras triangulares usadas en la técnica de conmutación PWM. El vector de estados está dado por las componentes directas $(d)$ y en cuadratura $(q)$ de las corrientes trifásicas, así como de la tensión en el bus de CD $\left[i_{1}{ }^{d}, i_{1} q, i_{2}{ }^{d}, i_{2} q, v_{c d}\right]^{T}$; y las entradas de control por las señales moduladoras $\left[m_{1}^{d}, m_{1}{ }^{q}, m_{2}{ }^{d}, m_{2}{ }^{q}\right]^{T}$. A partir de (4)-(8) se observa la naturaleza no lineal del convertidor BTB dada por la multiplicación de las entradas de control y las variables de estado, así como términos acoplados debidos a $\omega_{1,2} i_{1,2}^{d}$ y $\omega_{1,2} i_{1,2}^{q}$. Además, se definen las siguientes relaciones:

$$
P_{1,2}=\frac{3}{2}\left(V_{1,2}^{d} I_{1,2}^{d}+V_{1,2}^{d} I_{1,2}^{d}\right), Q_{1,2}=\frac{3}{2}\left(V_{1,2}^{q} I_{1,2}^{d}-V_{1,2}^{d} I_{1,2}^{q}\right)
$$

de donde se observa que a partir del control de las componentes de corriente $D Q$ es posible controlar el flujo el potencia, ya que los términos $D Q$ de la tensión se consideran constantes. Para el manejo del flujo de potencia en convertidores BTB los objetivos de control se pueden clasificar en tres de la siguiente manera: a) regulación de potencia activa, b) regulación de potencia reactiva y c) regulación de la tensión en el bus de CD. Está última es necesaria para mantener el balance de potencias entre VSC $_{1}$ y VSC 2 . Para simplificar la ley de control es habitual que un VSC controle el nivel de tensión de CD y otro la potencia activa; la regulación de potencia reactiva se atribuye al lado de CA correspondiente. En este trabajo $\mathrm{VSC}_{1}$ regula la tensión en el bus de CD y VSC 2 la potencia activa. Además, para explotar al máximo el uso del convertidor BTB dentro de la región lineal de operación (en donde se garantiza que la distorsión armónica total en corriente es mínima) es necesario determinar las cotas de potencia activa y reactiva en las cuales el convertidor BTB es capaz de operar. La región de la potencia activa es simétrica, sin embargo, para la potencia reactiva la región de operación inductiva es mayor que la capacitiva. El análisis de la región lineal 
de operación para convertidores BTB se presenta con mayor detalle en (Alcalá et al., 2010). Con los parámetros dados en la Tabla 1, se tiene que la potencia activa máxima que el convertidor BTB puede transferir está limitada por el inversor de menor capacidad (lo cual depende de la tensión de la red y de la inductancia de línea). De esta manera, la potencia activa máxima que el convertidor BTB puede transferir es de $17 \mathrm{~kW}$ y viene dictada por el $\mathrm{VSC}_{2}$. Por otro lado, las cotas de potencia reactiva están dadas por -2.5 $\mathrm{kVAR}<\mathrm{Q}_{1}<41.2 \mathrm{kVAR}$ para $\mathrm{VSC}_{1}$ y por $-2.0 \mathrm{kVAR}<\mathrm{Q}_{2}<31.9 \mathrm{kVAR}$ para $\mathrm{VSC}_{2}$.

Tabla 1: Parámetros del sistema

\begin{tabular}{|l|l|}
\hline Parámetros & Valor \\
\hline$V_{1,2}$ & $100 \mathrm{Vrms}$ \\
\hline$\omega_{1,2}$ & $377 \mathrm{r} / \mathrm{s}$ \\
\hline$L_{1}$ & $4.1 \mathrm{mH}$ \\
\hline$L_{2}$ & $5.3 \mathrm{mH}$ \\
\hline$R_{1}$ & $284 \mathrm{~m} \Omega$ \\
\hline$R_{2}$ & $330 \mathrm{~m} \Omega$ \\
\hline$V_{c d}$ & $320 \mathrm{~V}$ \\
\hline$S$ & $3 \mathrm{kVA}$ \\
\hline$f_{s w}$ & $4.8 \mathrm{kHz}$ \\
\hline$f_{\text {muestreo }}$ & $10 \mathrm{kHz}$ \\
\hline$U_{T 1,2}$ & $5 \mathrm{~V}$ \\
\hline$C_{c d}$ & $1050 \mu \mathrm{F}$ \\
\hline
\end{tabular}

\section{ESTRATEGIA DE CONTROL}

El sistema propuesto en lazo cerrado se muestra en la Fig. 3, en donde el convertidor se conecta a la línea a través de dos transformadores $(\Delta / \mathrm{Y})$. EI PLL (Phase-Locked Loop) se utiliza para sincronizar el convertidor con la frecuencia de la línea, así como para obtener el ángulo $\beta$, usado para calcular la transformación $D Q$. En estado estacionario $\beta_{1}$ está en fase con la fundamental de la fase A. Se utiliza únicamente un PLL, ya que ambos sistemas de CA operan a la frecuencia de línea. El bloque de control se basa en un control en cascada que incluye lazos de control interno y externo. Los lazos de control interno se usan como lazo principal y determinan las acciones de conmutación de $\mathrm{VSC}_{1}$ y $\mathrm{VSC}_{2}$. El objetivo de los lazos de control externos es suprimir el efecto debido a incertidumbres y/o perturbaciones en las referencias que utilizan los controladores internos. El controlador del lazo de tensión debe mantener la tensión de CD estable y regulada ante cualquier cambio de carga. Por otro lado, el control de potencia activa debe entregar la referencia de potencia deseada (que indica tanto la magnitud como el sentido). En cuanto a la potencia reactiva, la regulación depende del lado de CA correspondiente, de aquí que haya dos lazos externos para controlar la potencia reactiva asociada a $\mathrm{VSC}_{1}$ y $\mathrm{VSC}_{2}$.

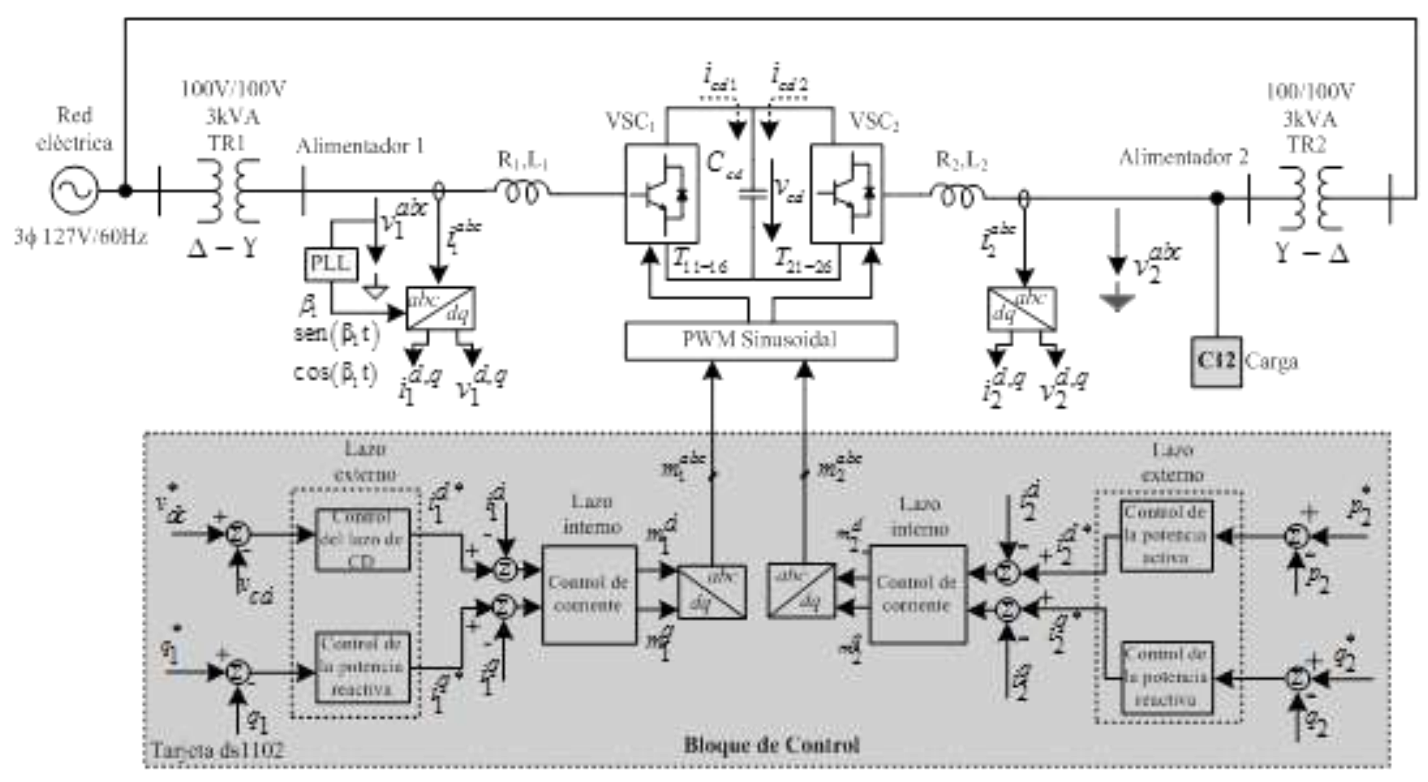

Fig. 3. Sistema de estudio propuesto. 
En este sentido, para lograr los objetivos de control requeridos por el sistema para el manejo del flujo de potencia es necesario cancelar los acoplamientos que existen entre las componentes activa y reactiva de la corriente, así como las no linealidades del sistema. El control de los lazos internos y externos se realiza utilizando controladores clásicos PI más un conjunto de términos que cancelan las no linealidades y acoplamientos a nivel local, lo que permite un control independiente de la potencia activa y la potencia reactiva en el punto de operación deseado. El control está dado por (10) y (11).

$$
\begin{aligned}
& m_{1}^{d}=k_{p 11}\left(i_{1}^{d^{*}}-i_{1}^{d}\right)+k_{i 11} \int\left(i_{1}^{d^{*}}-i_{1}^{d}\right) d t+\frac{2 U_{T 1} V_{1}^{d}}{v_{c d}^{*}}+\omega_{1} i_{1}^{q} \frac{U_{T 1} L_{1}}{v_{c d}^{*}} \\
& m_{1}^{q}=k_{p 12}\left(i_{1}^{q^{*}}-i_{1}^{q}\right)+k_{i 12} \int\left(i_{1}^{q^{*}}-i_{1}^{q}\right) d t+\frac{2 U_{T 1} V_{1}^{q}}{v_{c d}^{*}}-\omega_{1} i_{1}^{d} \frac{U_{T 1} L_{1}}{v_{c d}^{*}}
\end{aligned}
$$

Las leyes de control de corriente para $\mathrm{VSC}_{2}$ se obtienen bajo el mismo principio. Los lazos externos son los encargados de generar las referencias $\left(i_{1}^{d^{*}}, i_{1}^{q^{*}}, i_{2}^{d^{*}}, i_{2}^{q^{*}}\right)$ de los lazos internos de corriente. Las referencias de los lazos externos se calculan en tiempo real midiendo las tensiones y corrientes de línea en el marco de referencia abc de ambos VSC.

Las leyes de control de corriente para $\mathrm{VSC}_{2}$ se obtienen bajo el mismo principio. Los lazos externos son los encargados de generar las referencias $\left(i_{1}^{d^{*}}, i_{1}^{q^{*}}, i_{2}^{d^{*}}, i_{2}^{q^{*}}\right)$ de los lazos internos de corriente. Las referencias de los lazos externos se calculan en tiempo real midiendo las tensiones y corrientes de línea en el marco de referencia abc de ambos VSC; las funciones de transferencia están dadas por:

$$
\begin{aligned}
& H_{1}^{d}(s)=\frac{v_{c d}}{v_{c d}^{*}}=\frac{3 k_{p 12} v_{I}^{d} s+3 k_{i 12} v_{I}^{d}}{2 C_{c d} v_{d c}^{*} s^{2}+k_{p 12} v_{I}^{d} s+k_{i 12} v_{1}^{d}} \\
& H_{1,2}^{q}(s)=\frac{q_{1,2}}{q_{1,2}^{*}}=-\frac{s\left(k_{p 13} v_{1}^{d}-v_{1}^{q} i_{1}^{d}\right)-k_{i 13} v_{1}^{d}}{s\left(\frac{2}{3}-k_{p 13} v_{1}^{d}-v_{1}^{q} i_{1}^{d}\right)-k_{i 13} v_{1}^{d}} \\
& H_{2}^{d}(s)=\frac{p_{2}}{p_{2}^{*}}=-\frac{s\left(k_{p 21} v_{2}^{d}+v_{2}^{q} i_{2}^{d}\right)+k_{i 21} v_{1}^{d}}{s\left(\frac{2}{3}+k_{p 21} v_{2}^{d}+v_{2}^{q} i_{2}^{d}\right)+k_{i 21} v_{2}^{d}}
\end{aligned}
$$

El diseño de los controladores de los lazos externos se desacoplan de los lazos internos situándolos al menos una década abajo de la frecuencia de corte de los lazos internos el ancho de banda para éstos se sitúa en $20 \mathrm{~Hz}$.

\section{SIMULACIÓN}

Las simulaciones se realizaron bajo las plataformas de Matlab/Simulink ${ }^{\circ}$ y PSIM ${ }^{\circledR}$. Para mostrar el desempeño del convertidor BTB como controlador del flujo de potencia, se evalúan los siguientes casos:

\section{A. Caso 1. Regulación de potencia activa.}

Se asume que la carga mostrada en la Fig. $3\left(\mathrm{C}_{12}\right)$, demanda $3 \mathrm{~kW}$, los cuales son suministrados por TR1 a través del BTB y que en $0.2 \mathrm{~s}$ se invierte completamente el flujo de potencia, tal que ahora la carga inyecta potencia a TR1; los resultados se muestran en la Fig. 4. Se observa que el convertidor BTB puede invertir completamente el flujo de potencia activa (ante condiciones de regeneración) y el flujo de potencia reactiva permanece estable aun durante el transitorio. La tensión en el bus de CD presenta un sobretiro del $12.5 \%$ respecto del valor nominal $(320 \mathrm{~V})$ durante el transitorio, ya que momentáneamente el capacitor debe suministrar la potencia requerida para mantener el balance de energías.

\section{B. Caso 2. Regulación de potencia reactiva.}

El objetivo de este caso de estudio es mostrar la capacidad de operación del BTB como compensador de potencia reactiva exclusivamente. En esta condición, se realizan dos escalones de carga reactiva. Para ello en $0.2 \mathrm{~s}$ se aplica un transitorio de carga conectando una carga $C_{11}$ (a TR1) de -2.5 kVAR (restricción obtenida de la región de operación para $\mathrm{VSC}_{1}$ ) y en $0.3 \mathrm{~s}$ se conecta una segunda carga C12 de 3 kVAR. En la Fig. 5 se muestran los resultados; se observa que el BTB puede operar como un compensador de potencia reactiva. En cuanto a la potencia activa, se aprecia que la potencia suministrada por $\mathrm{VSC}_{1}$ (Wentrada) no es cero, si no que cambia y se estabiliza alrededor de 200W; esto se debe a que VSC ${ }_{1}$ es quién asume todas las pérdidas de conmutación y conducción asociadas a la operación del convertidor BTB. 


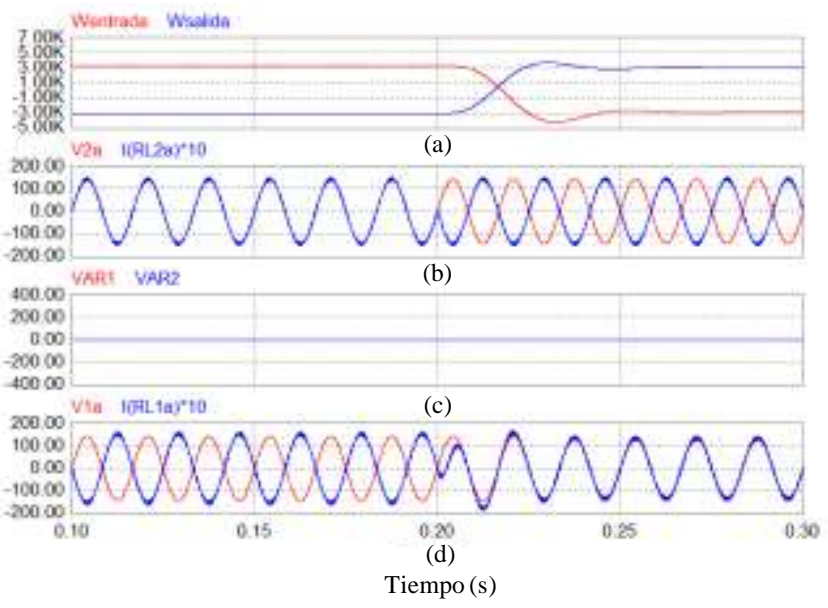

Fig. 4. Caso 1. (i) abc. (a) Potencia Activa (kW). (b). Tensión (V) y Corriente (A) para el $\mathrm{VSC}_{2}$. (c) Potencia Reactiva (VAR). (d) Tensión (V) y Corriente (A) para el $\mathrm{VSC}_{1}$.

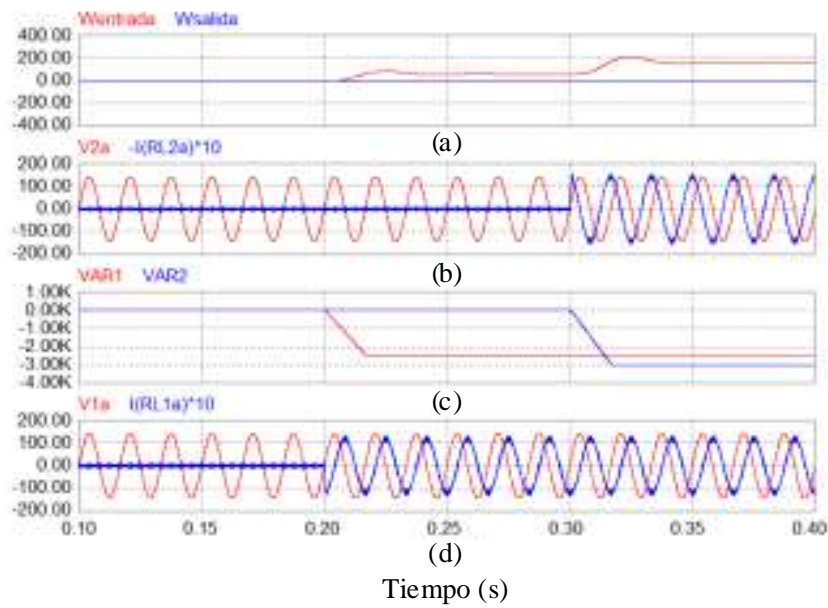

Fig. 5. Caso 2. (i) abc. (a) Potencia Activa (W). (b). Tensión (V) y Corriente (A) para el VSC 2 . (c)

Potencia Reactiva (VAR). (d) Tensión (V) y Corriente (A) para el $\mathrm{VSC}_{1}$.

\section{RESULTADOS EXPERIMENTALES}

El sistema de control propuesto se implementó usando la tarjeta ds 1102 de dSPACE $®$ operando a través de Matlab/Simulink ${ }^{\circledR}$ RTW. Para el esquema de potencia se desarrolló un prototipo BTB de 3 kVA, así como la circuitería necesaria para la generación del PWM y la adquisición de tensiones y corrientes. En la Fig. 6 se muestran las tensiones y corrientes de la fase a para $\mathrm{VSC}_{1}$ y $\mathrm{VSC}_{2}$ durante un transitorio en el cual se invierte completamente el flujo de potencia activa de $2 \mathrm{~kW}$ a $-2 \mathrm{~kW}$, se observa que las corrientes están desfasadas $180^{\circ}$ una respecto a la otra debido a que la potencia que entrega $\mathrm{VSC}_{1}$, la consume $\mathrm{VSC}_{2}$. En la Fig. 7 se muestran los resultados para un escalón de potencia reactiva. Inicialmente se transfieren $2 \mathrm{~kW}$ y se aplica un transitorio de carga en donde el BTB debe absorber -2.5kVAR a través de $\mathrm{VSC}_{2}$. De los resultados se puede corroborar que la transferencia de potencia activa no se afecta, incluso durante el transitorio, ya que tanto la tensión como la corriente de fase a permanecen estables. En la Fig. 8 se muestran la tensión y corriente de fase $a$ en $\mathrm{VSC}_{2}$, así como la componente activa de corriente y su referencia cuando se invierte el flujo de potencia activa de $2 \mathrm{~kW}$ a $-2 \mathrm{~kW}$. Se observa que la variable presenta un buen seguimiento hacia su referencia. La potencia se invierte en menos de un cuarto de ciclo de línea.

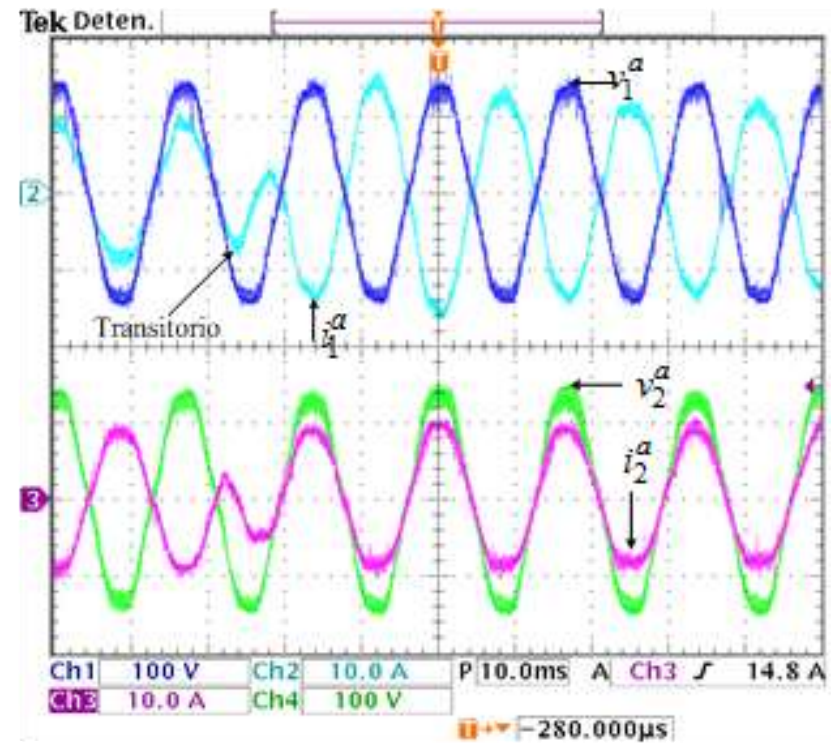

Fig. 6. Tensión-CH2, $\mathrm{CH} 4$ (100V/div) y corrientes$\mathrm{CH} 1, \mathrm{CH} 3\left(10 \mathrm{~A} /\right.$ div) de fase $a$ en los $\mathrm{VSC}_{1}$ (superior) y VSC 2 (inferior).

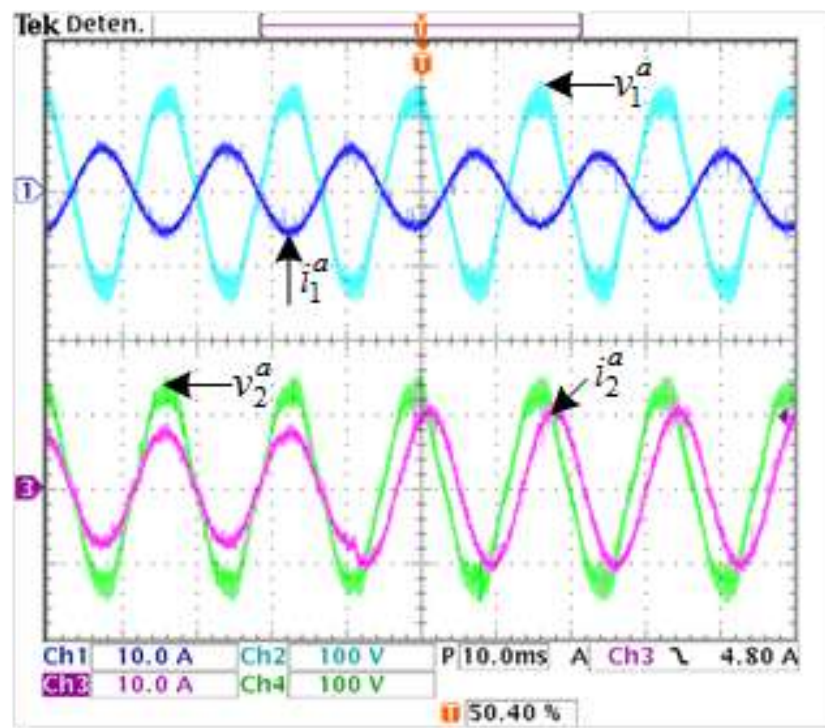

Fig. 7. Tensión-CH2, CH4 (100V/div) y corrientes$\mathrm{CH} 1, \mathrm{CH} 3(10 \mathrm{~A} / \mathrm{div})$ de fase a en los $\mathrm{VSC}_{1}$ (superior) y VSC 2 (inferior). 


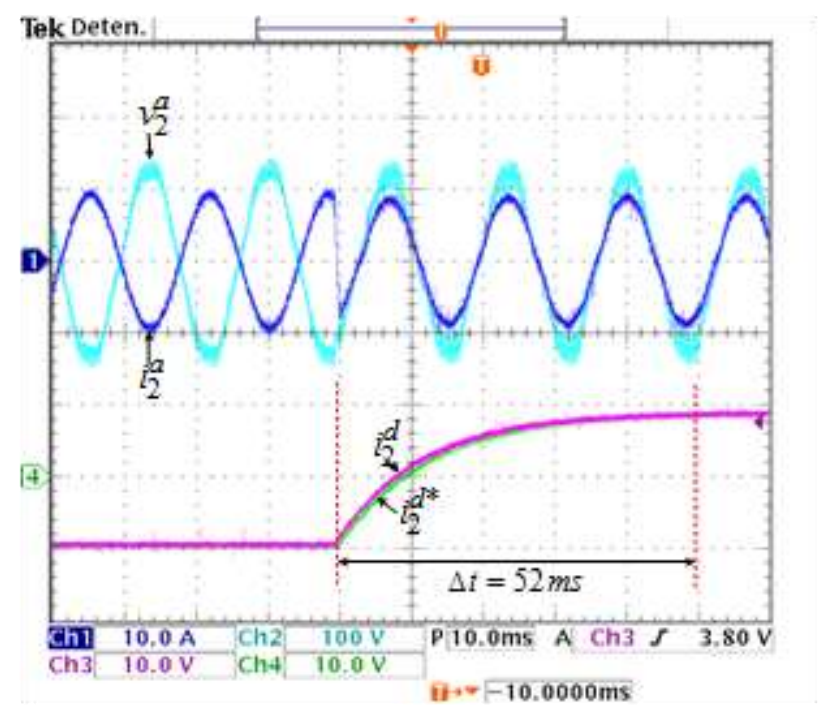

Fig. 8. Tensión $\mathrm{CH} 2, \mathrm{CH} 4$ (100V/div) y corrientes $\mathrm{CH} 1, \mathrm{CH} 3$ $\left(10 \mathrm{~A} /\right.$ div) de fase $a$ en $\mathrm{VSC}_{1}$ (superior) y $\mathrm{VSC}_{2}$ (inferior).

\section{CONCLUSIONES}

En este artículo se presentó un estudio del convertidor BTB para el manejo del flujo de potencia activa y reactiva. Para ello se estudian tres casos de operación en donde se muestra que el convertidor BTB tiene la capacidad de transferir bidireccionalmente potencia activa entre dos sistemas de CA interconectados, sin que la potencia reactiva se vea afectada. Así mismo, existe la capacidad de regular la potencia reactiva de una o varias cargas conectadas en sus terminales. La operación del convertidor BTB para el manejo del flujo de potencia se puede optimizar desde el punto de vista de electrónica de potencia considerando la región de operación lineal, y a partir de ella fijar las cotas máximas y mínimas de potencia activa y reactiva en las que el convertidor BTB puede operar sin entrar en la región de sobremodulación. Con ello, es posible mejorar el desempeño del convertidor y obtener un alto rendimiento en una relación-costo beneficio.

\section{REFERENCIAS}

Liming Liu; Pengcheng Zhu; Yong Kang; Jian Chen, Power-Flow Control Performance Analysis of a Unified Power-Flow Controller in a Novel Control Scheme. Power Delivery, IEEE Transactions on, ISSN: 0885-8977, 22(3), 1613, 1619..(2007)

Weihao Hu; Zhe Chen; Yue Wang; Zhaoan Wang, Flicker Mitigation by Active Power Control of VariableSpeed Wind Turbines With Full-Scale Back-to-Back Power Converters. IEEE Transactions on Energy Conversion, 24(3), 640,649. (2009).

Saeedifard, M.; Iravani, R., Dynamic Performance of a Modular Multilevel Back-to-Back HVDC. IEEE Transactions on Power Delivery System, ISSN: 0885-8977, 25(4), 2903, 2912 (2010)

Alcalá, J., V. Cárdenas, E. Rosas y C. Núñez. Control System Design for Bi-directional Power Transfer in Single - Phase Back - to - Back Converter Base on the Linear Operating Region. Proc. IEEE Applied Power Electronic Conference and Exposition, 1651-1658. (2010)

Majumder, R.; Ghosh, A; Ledwich, G.; Zare, F., Power Management and Power Flow Control With Back-toBack Converters in a Utility Connected Microgrid. IEEE Transactions on Power Systems, ISSN: 0885-8950, 25(2), 821,834 (2010).

Zeliang Shu; Shaofeng Xie; Qunzhan Li, Single-Phase Back-To-Back Converter for Active Power Balancing, Reactive Power Compensation, and Harmonic Filtering in Traction Power System. IEEE Transactions on Power Electronics, 26(2), 334,343 (2011).

Gallego-Gómez, Juan D, Cano-Quintero, Juan B and Muñoz-Galeano, Nicolás, Análisis de Flujos de Potencias Ineficientes Producidos por Convertidores de Potencia. Inf. tecnol., 25(3), 93-102 (2014).

Guacaneme, Javier A, Velasco, David and Trujillo, César L., Revisión De Las Características de Sistemas de Almacenamiento de Energía para Aplicaciones en Micro Redes. Inf. tecnol.25(2) 175-188 (2014). 